\title{
Decay dynamics of excitonic polarons in InAs/GaAs quantum dots
}

Cite as: J. Appl. Phys. 110, 074303 (2011); https://doi.org/10.1063/1.3639310

Submitted: 16 June 2011 . Accepted: 11 August 2011 . Published Online: 03 October 2011

S. Werner, J. S. Reparaz, M. R. Wagner, P. Zimmer, N. N. Ledentsov, J. Kabuss, M. R. Dachner, M. Richter, A. Knorr, C. Thomsen, and A. Hoffmann

\section{ARTICLES YOU MAY BE INTERESTED IN}

Invited Review Article: Single-photon sources and detectors

Review of Scientific Instruments 82, 071101 (2011); https://doi.org/10.1063/1.3610677

Phonon-assisted capture and intradot Auger relaxation in quantum dots

Applied Physics Letters 74, 2818 (1999); https://doi.org/10.1063/1.124024

Low-temperature photocarrier dynamics in monolayer $\mathrm{MoS}_{2}$

Applied Physics Letters 99, 102109 (2011); https://doi.org/10.1063/1.3636402

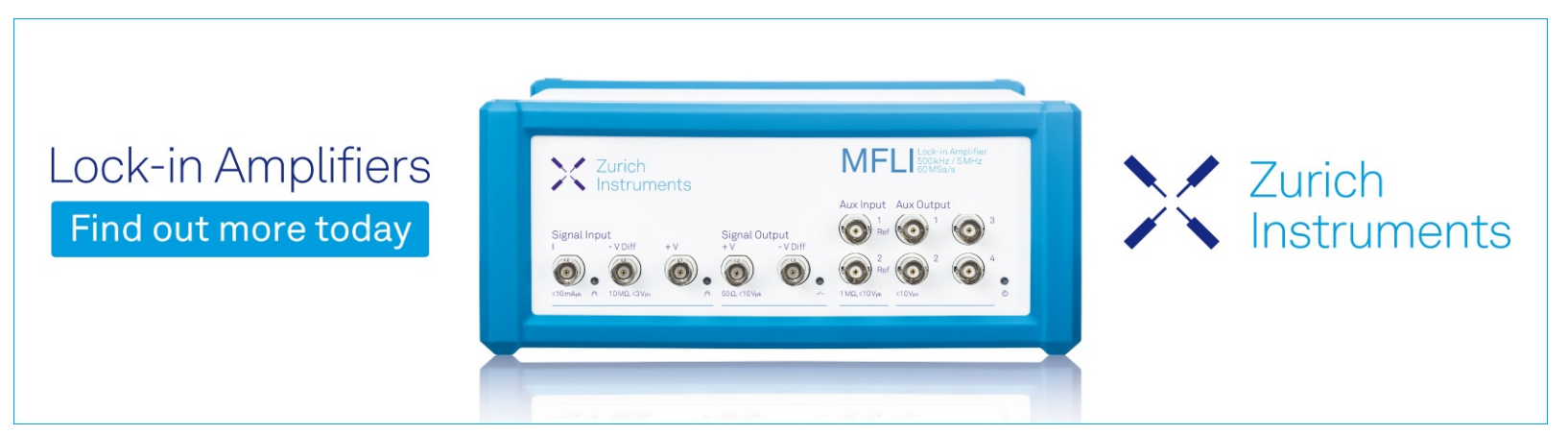




\title{
Decay dynamics of excitonic polarons in InAs/GaAs quantum dots
}

\author{
S. Werner, ${ }^{1}$ J. S. Reparaz, ${ }^{1}$ M. R. Wagner, ${ }^{1}$ P. Zimmer, ${ }^{1}$ N. N. Ledentsov, ${ }^{1}$ \\ J. Kabuss, ${ }^{2}$ M. R. Dachner, ${ }^{2}$ M. Richter, ${ }^{2}$ A. Knorr, ${ }^{2}$ C. Thomsen, ${ }^{1}$ and A. Hoffmann, ${ }^{1}$ \\ ${ }_{1}^{1}$ Institut für Festkörperphysik, Technische Universität Berlin, Hardenbergstr. 36, 10623 Berlin, Germany \\ ${ }^{2}$ Institut für Theoretische Physik, Nichtlineare Optik und Quantenelektronik, Technische Universität Berlin, \\ EW 7-1, Hardenbergstr. 36, D-10623 Berlin, Germany
}

(Received 16 June 2011; accepted 11 August 2011; published online 3 October 2011)

\begin{abstract}
We present time-resolved studies of the exciton-phonon interaction in self-assembled InAs/GaAs quantum dots. Different scattering and luminescence processes were investigated by time-resolved spectroscopy exciting resonantly into the quantum dot's electronic structure. By studying the characteristic decay times of the ground state and of several phonon-assisted recombinations we were able to distinguish a resonant Raman process from a phonon-assisted photoluminescence process which are always simultaneously present and can interfere with each other. While lifetimes under 30 ps were observed for the coherent Raman process, the incoherent phonon-assisted recombination exhibited typical lifetimes of around $1 \mathrm{~ns}$ independently of the excitation energy. We conclude that under resonant excitation the dominant radiative recombination process in this system always involves an electronic state of the ground state of the quantum dot's electronic structure. Combining temperature-dependent and time-resolved measurements we show that a weak phonon-bottleneck is present in the low temperature regime $(<130 \mathrm{~K})$, while it disappears for higher temperatures. (C) 2011 American Institute of Physics. [doi:10.1063/1.3639310]
\end{abstract}

\section{INTRODUCTION}

The potential applications of quantum dots (QDs) systems in solid-state laser devices have triggered great interest in the fundamental properties of these nanostructures. Some typical examples of their most prominent advantages compared to the bulk case are higher temperature stability, ${ }^{1}$ lower threshold current, ${ }^{2}$ and improved noise characteristics. $^{3}$ The physical origin of these desirable properties relies mostly on their $\delta$-function electronic density of states, which originates from the strong quantum confinement potential. However, the relaxation mechanisms of the excited carriers are strongly influenced by this discretization and, thus, the electronic states cannot couple to phonons as efficiently as in bulk materials. Consequently, the exciton-phonon interaction in self-organized semiconductor QDs has been subject of a great number of studies. ${ }^{4-8}$ Among others, this interaction influences the free-carrier relaxation as well as the recombination and dephasing dynamics of confined excitons. Because of the strong spatial localization, the overlap between the electronic and vibrational wavefunctions is fairly large, which greatly enhances the electron-phonon interaction. In addition, the resultant strong coupling between excitons and phonons leads to the formation of QD excitonic polarons. ${ }^{9-15}$ Regarding the optical emission of these low dimensional structures several contributions can be distinguished as shown schematically in Fig. 1. From the possible coherent processes the lowest order of light-matter interaction leads to elastic scattering of the incident light, commonly known as Rayleigh scattering as shown in Fig. 1(a), while in higher orders coherent inelastic scattering is dominated by Raman scattering [Fig. 1(b)]. For the latter, emission (Stokes) or absorption (anti-Stokes) of phonons is possible, which leads to a red or blueshift of the Rayleigh component, respectively. In addition, incoherent scattering processes are usually represented by luminescence processes [Fig. 1(c)]. In this case, the initial coherence is lost after a certain time interval by scattering with defects, boundaries or even phonons. In contrast to the previous coherent processes, in a luminescence process a real state (in which the initial coherence is lost) is essential. Furthermore, the initially excited states can recombine with the emission of one or several phonons, which is commonly referred to as phonon-assisted luminescence. The oscillator strength of this process is partly determined by the magnitude of the electron-phonon coupling. Nevertheless, it is not always the case that electronic excitations successfully couple to phonons. In some situations a suppressed carrier relaxation and restriction of the ground state (GS) population upon nonresonant excitation has led to the observation of a phononbottleneck. ${ }^{16-18}$ Due to the fact that many of these different contributions to the optical emission are energetically degenerated, in some situations it is almost impossible to distinguish them only by investigating their time-integrated optical emission.

Therefore, the purpose of this work is to show that by combining resonant excitation spectroscopy with timeresolved photoluminescence measurements we are able to elucidate the origin of the interaction of phonons and excitons in InAs/GaAs quantum dots. By investigating the lifetime of several optical transitions we distinguish their origin as arising from coherent Raman processes, or from incoherent phonon-assisted recombination. As a striking result, we show that the main radiative recombination mechanism is given by an outgoing resonance process involving the ground-state level of the QDs as active electronic states. Furthermore, through time-resolved and temperature-dependent 

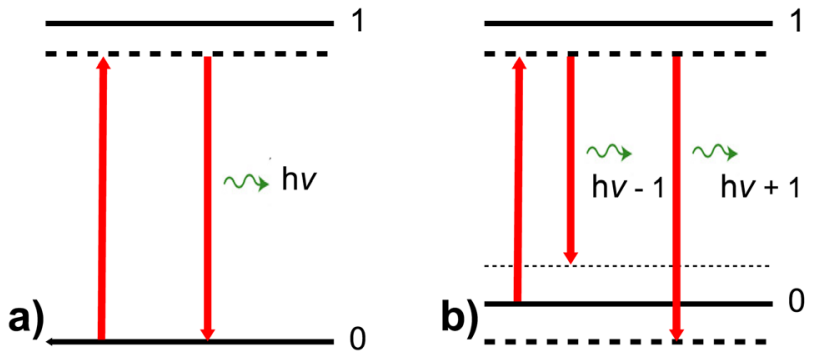

photoluminescence experiments we establish the existence of a weak phonon-bottleneck for temperatures below $130 \mathrm{~K}$.

\section{EXPERIMENTAL DETAILS}

The investigated sample was grown on a $\mathrm{GaAs}(100)$ substrate by molecular beam epitaxy (MBE) using the following growth sequence: GaAs substrate/300 nm GaAs/100 $\mathrm{nm} \mathrm{Al}_{0.3} \mathrm{Ga}_{0.7} \mathrm{As} / 100 \mathrm{~nm} \mathrm{GaAs} / 0.7 \mathrm{~nm}$ InAs QDs(2.3 ML)/ $0.6 \mathrm{~nm}$ AlAs cap layer(2.0 ML)/4 nm $\mathrm{In}_{0.25} \mathrm{Al}_{0.75} \mathrm{As} / 970 \mathrm{~nm}$ $\mathrm{GaAs} / 100 \mathrm{~nm} \mathrm{Al}{ }_{0.3} \mathrm{Ga}_{0.7} \mathrm{As} / 10 \mathrm{~nm}$ GaAs. Photoluminescence (PL) measurements were carried out in the range between 2 and $300 \mathrm{~K}$ using a $30 \mathrm{~cm}$ focal distance McPherson Monochromator with a spectral resolution below $1 \mathrm{meV}$. By achieving the lowest temperatures $(2 \mathrm{~K})$ the sample was immersed in a bath cryostat operated at superfluid helium temperature. For the higher range between 5 and $300 \mathrm{~K}$ we used a continuous-flow cryostat. Resonant PL spectra were excited by an optical parametric oscillator (OPO) tunable between $1.033 \mathrm{eV}(1.2 \mu \mathrm{m})$ and $1.240 \mathrm{eV}(1 \mu \mathrm{m})$, pumped by a Ti:sapphire (Ti:Sa) laser system and detected using an InGaAs diode. Time-resolved photoluminescence (TRPL) spectra were measured with a micro-channel plate (MCP S1) using the time-correlated single-photon counting technique, and the time resolution of the detection system is estimated to be around $30 \mathrm{ps}$. The temporal linewidth of the laser pulses was $1.4 \mathrm{ps}$ at a repetition rate of $80 \mathrm{MHz}$, and typical laser powers were around $150 \mathrm{~mW}$.

\section{RESULTS AND DISCUSSION}

\section{A. Steady-state photoluminescence spectra}

In order to investigate the influence of the electronphonon coupling on the recombination processes of the optically excited electronic states, we have chosen an InAs QDs sample where the first excited state is energetically located about + 3LO phonon energies away from the GS energy position. Typical PL spectra for non-resonant $(1.6 \mathrm{eV})$ and resonant excitation $(1.158 \mathrm{eV})$ at $2 \mathrm{~K}$ are shown in Fig. 2(a). The dashed line in the spectra corresponds to the nonresonant excitation case $(1.60 \mathrm{eV})$ above the GaAs barrier bandgap $\left(E_{\mathrm{GaAs}} \approx 1.52 \mathrm{eV}\right)$. With this excitation energy we obtain an overview spectrum of the sample showing that the maximum GS emission of the QDs ensemble is centered around $1.055 \mathrm{eV}$, while the corresponding first excited state is observed at $1.15 \mathrm{eV}$. Thus, the energy splitting between the GS and the first excited state is about $95 \mathrm{meV}$. In addition, a full-width at half-maximum (FWHM) of $50 \mathrm{meV}$ is

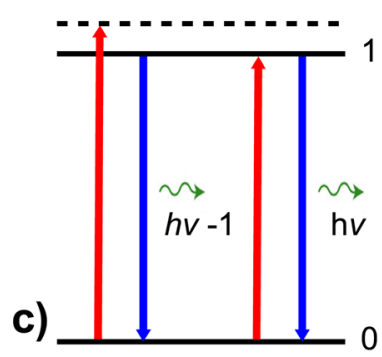

FIG. 1. (Color online) Schematic diagram showing different light scattering processes. (a) Elastic Rayleigh process. (b) Inelastic Stokes and anti-Stokes Raman process. (c) Phonon-assisted photoluminescence processes. Outgoing (left) and incoming (right) resonant cases. obtained for the GS, reflecting the broad size distribution of the QDs ensemble which overcame through the resonant excitation conditions, i.e., only a sub-ensemble of QDs is excited. The solid line spectrum in Fig. 2(a) represents a typical PL spectrum under resonant excitation with $1.158 \mathrm{eV}$. Under these conditions the spectrum changes tremendously in comparison to the non-resonant case. The narrow line on the high energy side of this spectrum corresponds to the laser line (Rayleigh component), which is resonant with the first excited state of the QDs whose GS lies around $1.055 \mathrm{eV}$.

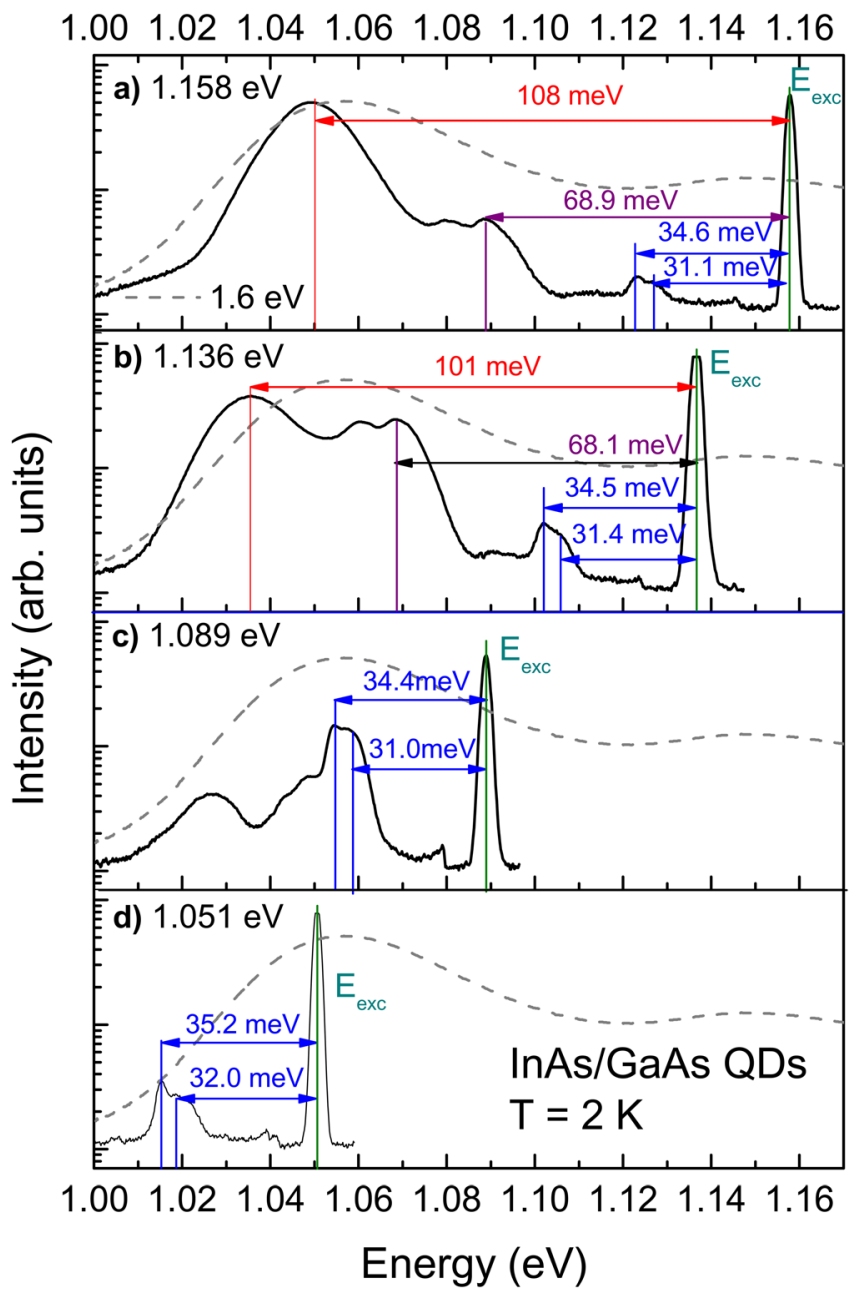

FIG. 2. (Color online) Photoluminescence spectra at $2 \mathrm{~K}$ of InAs QDs for different resonant excitation energies of (a) $1.158 \mathrm{eV}$, (b) $1.136 \mathrm{eV}$, (c) $1.089 \mathrm{eV}$, and (d) $1.051 \mathrm{eV}$. The arrows display the energy separation of the first (1LO) and second (2LO) order optical phonons. The dashed lines account for the non-resonant excitation case $\left(E_{\mathrm{exc}}=1.6 \mathrm{eV}\right)$. 
Moreover, it could also resonate with a smaller number of comparatively smaller QDs whose GS lies above $1.055 \mathrm{eV}$. The emission at $1.05 \mathrm{eV}$ exhibits a FWHM of $25 \mathrm{meV}$, which is significantly smaller in comparison to that observed for non-resonant excitation. Although, the attribution of this transition to the GS recombination is extremely tempting, several other possible candidates should also be considered. The possible processes that could be responsible for this transition are (i) a $3 \mathrm{LO}$ phonon-assisted recombination process of the first excited state (resonant with the laser excitation) of a certain sub-ensemble of QDs, (ii) a $3 \mathrm{LO}$ phonon-assisted process of the GS of comparatively smaller QDs which energy position coincides with the laser excitation, (iii) a $3 \mathrm{LO}$ outgoing resonance process in which the active electronic states are always the GS of a certain subensemble of QDs. The difficulty in ruling out any of these candidates relies partly due to the energy separation between the GS and the first excited state equal to the energy of a 3LO process. Concerning (i), Fig. 2(c) shows that although the laser excitation energy is well below any of the first excited levels, a strong emission around $1.025 \mathrm{eV}$ resembling that at $1.05 \mathrm{eV}$ in Fig. 2(a) is clearly present. The case described in (ii) is also relatively unlikely to be the correct assignment since the amount of QDs which have their ground state in this excitation range is almost negligible. Nevertheless, due to the strong enhancement of the scattering cross section originating from the resonant excitation, none of these candidates can be fully ruled out based exclusively on steady-state PL measurements. As we will show in the next section, it is only through time-resolved investigations that we are actually able to identify (iii) the process responsible for this transition.

Several other transitions are also observed in Fig. 2, e.g., a double peak at 1.129 and $1.125 \mathrm{eV}$ in Fig. 2(a) corresponding to 31.1 and $34.6 \mathrm{meV}$ below the laser excitation, respectively. The peak located at $31.1 \mathrm{meV}$ from the laser line is generally attributed to an InAs/GaAs QD LO phonon, ${ }^{19}$ while that at $34.6 \mathrm{meV}$ probably arises from an interface vibrational mode of the QDs as discussed in Ref. 19. The peaks located around $40 \mathrm{meV}$ below the laser energy probably originates from $1 \mathrm{LO}$ modes of the AlAs cap layer. Additionally, Fig. 2(a) shows a second double structure at 1.091 and $1.082 \mathrm{eV}$, whose energy distances to the excitation laser are 68.9 and $77.8 \mathrm{meV}$, respectively. These peaks correspond to $2 \mathrm{LO}$ processes of the transitions already discussed. The 2LO mode around $68.9 \mathrm{meV}$ (see arrows in Fig. 2) corresponds to the 31.1 and $34.6 \mathrm{meV} 1 \mathrm{LO}$ modes, while that around $77.8 \mathrm{meV}$ corresponds to the AlAs modes around $40 \mathrm{meV}$ from the laser excitation. We remark that solely those phonons arising from the QDs will resonate with the electronic levels of the QDs as varying the excitation frequency. Thus, we focus the reader's attention to the $1 \mathrm{LO}$ modes at 31.1 and $34.6 \mathrm{meV}$, and their $2 \mathrm{LO}$ mode around $68.9 \mathrm{meV}$ (marked with horizontal arrows in Fig. 2). Finally, the electronic states involved in these phonon-assisted processes cannot be identified solely from the PL spectra and, consequently, the different scenarios (i), (ii), and (iii) already discussed for the origin of the $1.05 \mathrm{eV}$ emission in Fig. 2(a) are also valid for these phonon-assisted transitions.
In Fig. 3 we show the different transitions of the spectra in Fig. 2 as a function of the excitation energy. While the $1 \mathrm{LO}$ and $2 \mathrm{LO}$ shift almost parallel to the laser (also shown in the Fig. 3 for comparison), the GS exhibits a highly nonparallel shift. This result represents a direct proof of the fact that the $1 \mathrm{LO}$ and 2LO emissions are phonon modes, while it suggests that the peak labeled as GS is mainly dominated by the emission of the GS itself, with a smaller contribution from a $3 \mathrm{LO}$ process. The energy position of the GS depends on the laser excitation, since for each excitation energy a different QDs sub-ensemble is selected. This result is further confirmed by the photoluminescence excitation spectrum shown in the inset to Fig. 3. The detection energy was set to the GS and the excitation was continuously increased to higher energies. It is apparent that the different phonon modes act as efficient recombination channels for the GS. This behavior is similar to the observation of Heitz et al. ${ }^{15}$ and influences significantly the dynamical properties of the InAs QDs.

Additionally, we have investigated the temperature dependence of these transitions for different excitation energies. We show in Fig. 4 a temperature series between 5 and $300 \mathrm{~K}$ for the case of $1.136 \mathrm{eV}$ laser excitation, corresponding to the spectrum of Fig. 2(b). We have chosen this case as a representative example since for this excitation the 1LO, 2LO, and GS emissions can be simultaneously observed with maximum relative intensity. Regarding the intensity of the different transitions a fundamental difference is observed between the GS and 1LO/2LO emissions. While the intensity of the GS increases toward a maximum at around $130 \mathrm{~K}$, the intensity of $1 \mathrm{LO} / 2 \mathrm{LO}$ monotonically decreases with increasing temperature as shown in the inset to Fig. 4. The intensity increase of the GS can be explained by considering the occurrence of a phonon-bottleneck for low temperatures. When excited resonantly into the first excited state, the carrier relaxation to the GS through the emission of several phonons is partly suppressed at low temperatures. As temperature increases this suppression is abrogated and the

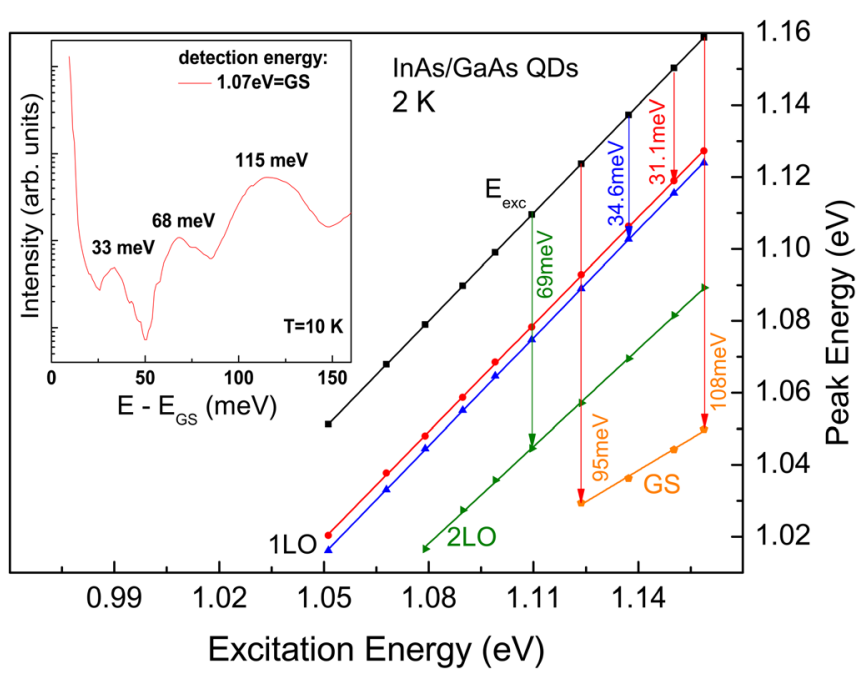

FIG. 3. (Color online) Energy position of the maximum intensity of the transitions of Fig. 2 as a function of the excitation energy. In the inset, a photoluminescence excitation spectrum (PLE) with the detection energy set to the GS position. 


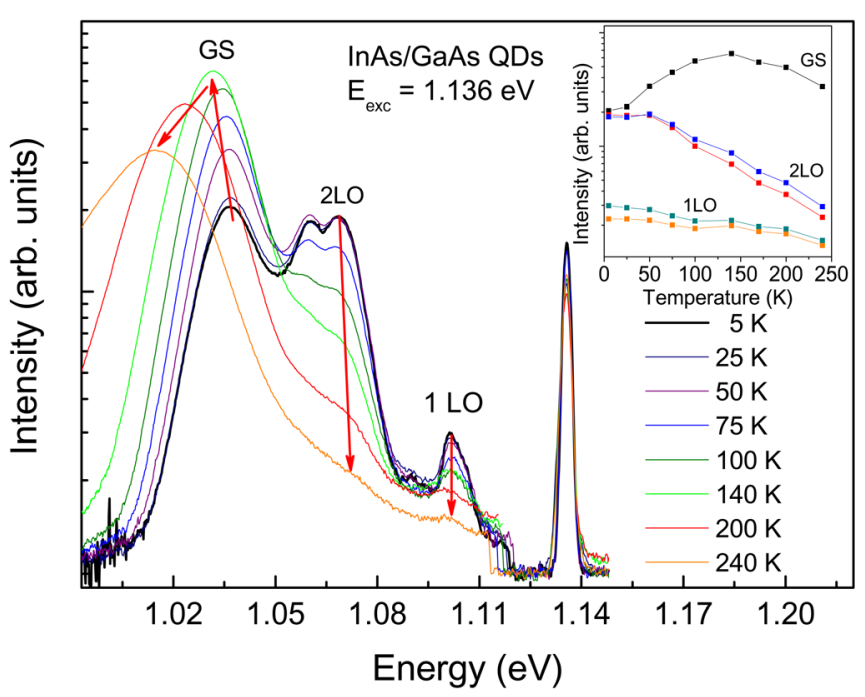

FIG. 4. (Color online) Photoluminescence spectra for resonant excitation $\left(E_{\text {exc }}=1.136 \mathrm{eV}\right)$ at different temperatures. In the inset, the maximum intensity of the 1LO, 2LO, and GS are shown as a function of temperature.

excited electrons can be successfully transferred to the GS via the emission of optical phonons. In addition, as temperature increases the GS shifts to lower energies following a Varshni behavior (not shown), while the energy position of the $1 \mathrm{LO} / 2 \mathrm{LO}$ remains approximately unchanged.

\section{B. Time-resolved photoluminescence}

In this section we investigate the dynamics of each of the transitions presented in the previous part of this work. The dynamics of these transitions turn out to be essential to understand the origin of the electron-phonon interaction in this system. We will focus on resonant excitation, which is responsible for the great variety of optical emissions observed in the PL spectra of Fig. 2. In Fig. 5 we show the resonant PL spectrum at $2 \mathrm{~K}$ for the case of resonant excitation $\left(E_{\mathrm{exc}}=1.158 \mathrm{eV}\right)$ with the first excited state in the maxi-

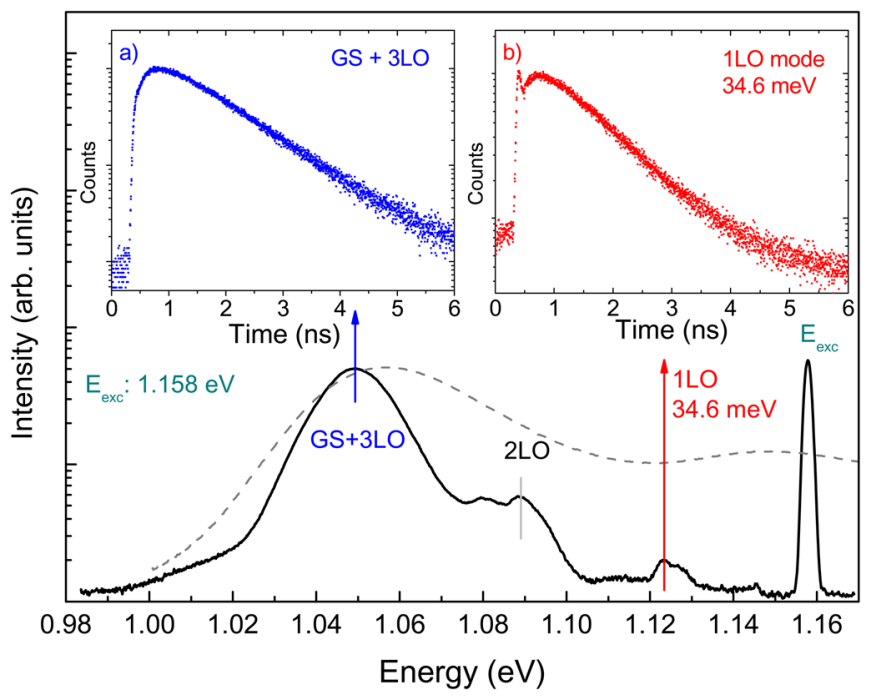

FIG. 5. (Color online) Steady-state photoluminescence spectrum for resonant excitation $\left(E_{\text {exc }}=1.158 \mathrm{eV}\right)$. The insets show the dynamical response detected at (a) the GS + 3LO energy and, (b) the LO at $34.6 \mathrm{meV}$ at $2 \mathrm{~K}$. The vertical arrows indicate the energy position of each transient. mum of the size distribution which is approximately proportional to the intensity of the PL spectrum under nonresonant excitation (see dashed line spectrum in Fig. 5). As we have previously discussed, this represents an extremely particular excitation condition since the energy difference between the first excited state and the GS equals the energy of a $3 \mathrm{LO}$ process and, thus, the initially excited electronic states can be scattered to the GS through the emission of three LO phonons. In Fig. 5 we observe three main transitions around $1.25 \mathrm{eV}$ (1LO), $1.09 \mathrm{eV}$ (2LO), and $1.05 \mathrm{eV}$ $(\mathrm{GS}+3 \mathrm{LO})$. Figure 5(a) shows the decay dynamics for the transition around $1.05 \mathrm{eV}$. All the transients were fitted by performing a multi-exponential deconvolution procedure of the measured transients from the system response characteristics. For the $1.05 \mathrm{eV}$ transition we used two single exponential forms that account for the observed rise and decay times, which resulted in $\tau_{r}=270 \mathrm{ps}$ and $\tau_{d 1}=1 \mathrm{~ns}$, respectively. In Fig. 5(b) we show the transient of the 1LO transition around $1.25 \mathrm{eV}$. In this case an additional exponential form was included into the fitting model which accounts for the observed short decay component $\left(\tau_{d 2}\right)$. We obtained $\tau_{r}=300 \mathrm{ps}, \tau_{d 1}=1 \mathrm{~ns}$, and $\tau_{d 2} \leq 30 \mathrm{ps}$ for the rise, long, and short decay times, respectively. Concerning $\tau_{d 2}$, we provide only a maximum value since this is below the time resolution of the experimental setup ( $\approx 30 \mathrm{ps}$ ). The main difference between the decay times of these two transitions is the additional short component $\tau_{d 2}$ observed for the $1 \mathrm{LO}$, which is only and always observed at an energy distance of 1LO ( $\approx 34.4 \mathrm{meV}$ ) from the laser excitation. We attribute this emission to a coherent Raman process originating from the whole dot distribution. We made a similar observation for InGaAs/GaAs quantum dots. ${ }^{20}$ However, it is very likely that this transition is dominated by those QDs fulfilling the outgoing resonance condition, since in this case the Raman cross section is greatly enhanced. To further support this assignment, we have performed theoretical calculations. A microscopic equation of motion approach was chosen using the density-matrix formalism within a higher order Bornapproximation for quantum emissions assisted by a single phonon process. A detailed description of the theoretical model used for the calculations can be found elsewhere. ${ }^{21}$ In Fig. 6 we show a measured PL transient of the 1LO transition at $75 \mathrm{~K}$ for $1.136 \mathrm{eV}$ of laser excitation together with the calculations. The agreement between the theoretical and experimental behavior is shown to be quite satisfactory, i.e., the Raman (coherent, $\tau_{d 2}$ ) and the phonon-assisted luminescence process (incoherent, $\tau_{d 1}$ ) can be separated through their different temporal behavior.

The origin of the long decay component $\left(\tau_{d 1}\right)$ can be revealed by studying the energy dependence of the lifetimes of the 1LO transition. In Table I we show the values for $\tau_{d 1}$ and $\tau_{d 2}$ as resulting from the fits to the transients for different excitations as well as their intensity ratios. The fact that $\tau_{d 1}$ is almost independent on the excitation energy constitutes a striking result since it suggests that this transition originates from recombination of the GS of the QDs independently on the excitation mechanism. When the laser energy is set to the lowest value of $1.059 \mathrm{eV}$, only excitation of the GS of the QDs system is possible. In this case we obtain $\tau_{d 1}=1.3 \mathrm{~ns}$, 


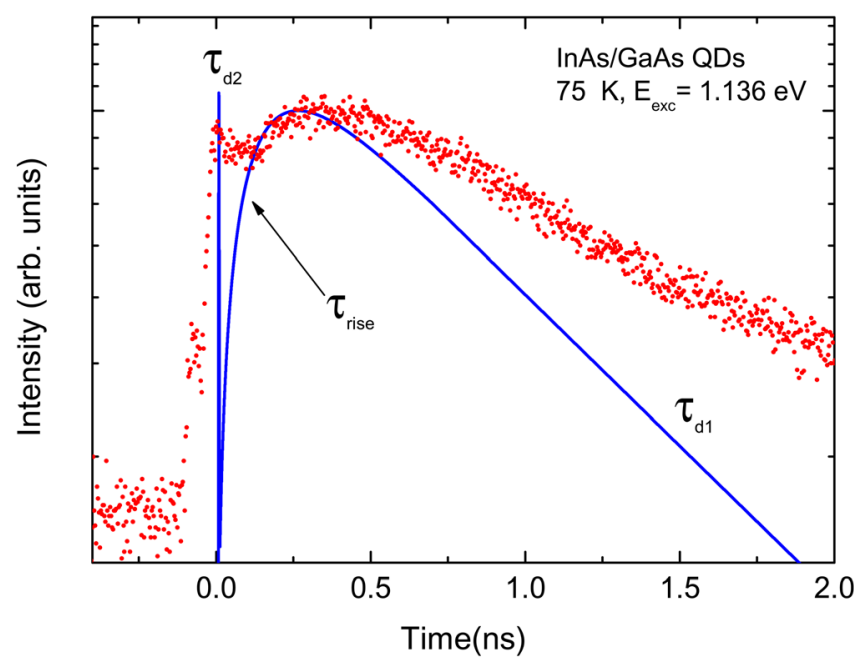

FIG. 6. (Color online) Comparison of the measured transient for the 1LO phonon transition at $34.6 \mathrm{meV}$ with theoretical calculations. The laser excitation was set to $1.136 \mathrm{eV}$ and the temperature to $75 \mathrm{~K}$. The red dots represent the experimental data, while the solid blue line accounts for the calculated incoherent $\left(\tau_{d 1}\right)$ and coherent $\left(\tau_{d 2}\right)$ components.

which constitutes a typical lifetime for the GS emission of these QDs. Table I shows that as the excitation energy increases, $\tau_{d 1}$ decreases only $30 \%$ over the whole energy range. This tendency can be understood considering that for the higher excitation energies a sub-ensemble of smaller QDs is selected for which the electron and hole wavefunctions have a larger overlap. This leads to a larger oscillator strength (smaller lifetimes) for the electron-hole recombination. Furthermore, for the larger excitation energies no additional decay component to the measured lifetime is observed, which could arise from recombination of the first excited state. Typically, the lifetime of this transition is expected to be far smaller than that observed for the GS. Thereto, independently of the particular laser excitation, the long decay component $\tau_{d 1} \approx 1$ ns originates from an electronic state of the GS of the QDs electronic structure, which

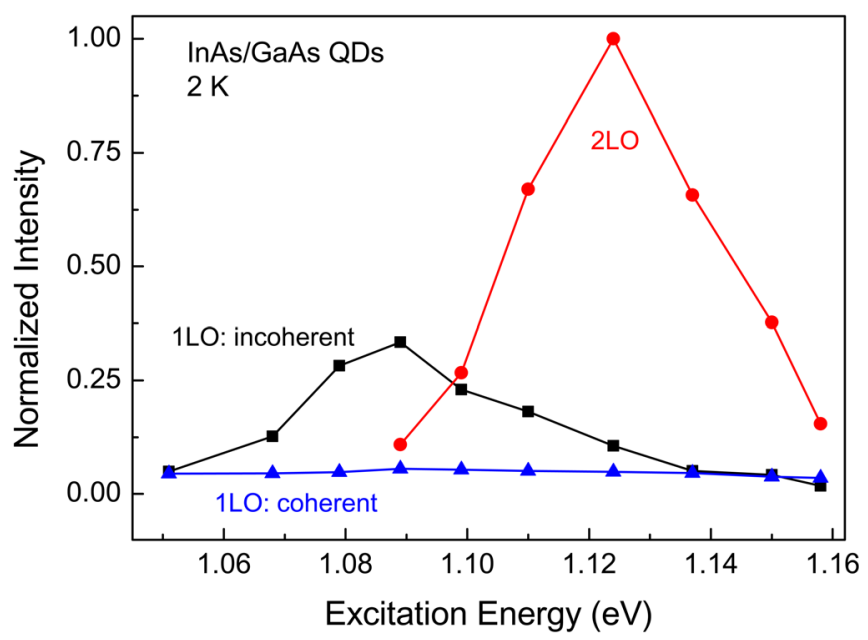

FIG. 7. (Color online) Intensity of the LO (black quadrangles) and 2LO (red circles) signals for varying excitation energies. In addition, the fraction of the LO signal assigned to the Raman scattering has been estimated and is shown by the blue line with triangles.

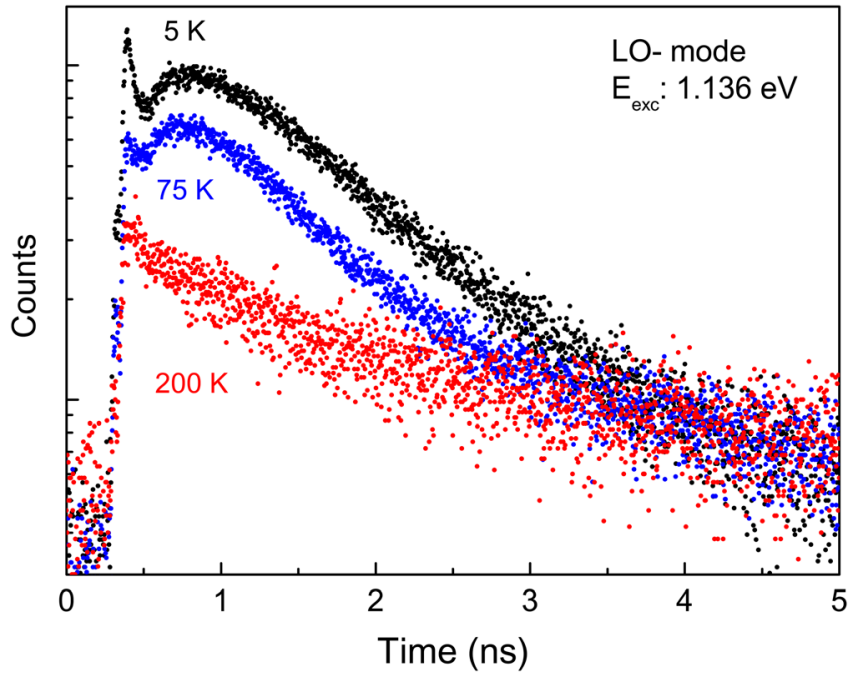

FIG. 8. (Color online) Decay dynamics of the LO mode for $1.136 \mathrm{eV}$ laser excitation and for different temperatures of 5, 75, and $200 \mathrm{~K}$.

fulfills the outgoing resonance condition with the laser excitation energy.

In Fig. 7 we show the intensity resonance profiles for the different decay components of the $1 \mathrm{LO}$ and $2 \mathrm{LO}$ modes. We remark that deconvolution of the intensity contributions corresponding to $\tau_{d 1}$ and $\tau_{d 2}$ is only possible to achieve through the time-selectivity of the time-resolved measurements. This would be impossible to accomplish based solely on an energy-dispersive spectroscopic measurement since these transitions are energetically degenerated. As the excitation energy increases, the intensity of the $1 \mathrm{LO}$ also increases upon reaching a maximum at an excitation of $1.089 \mathrm{eV}$, which equals the energy of the 1LO above the maximum intensity of the GS. It is interesting to note that a strong resonance is exclusively observed for the outgoing resonance case $\left(E_{\mathrm{exc}}=E_{\mathrm{GS}}+E_{1 \mathrm{LO}}\right)$, whereas no resonance is observed for the incoming case $\left(E_{\mathrm{exc}}=E_{\mathrm{GS}}\right)$. A comparable behavior was observed for the $2 \mathrm{LO}$, which showed a resonant behavior at about the energy of the 2LO from the GS emission. In addition, the energy dependence of the relative intensity of the coherent Raman process does not exhibit any resonance for the whole range. Nevertheless, if the resonance condition is lost no Raman contribution is observed. This supports our previous assumption that the Raman component mainly originated from those QDs fulfilling the outgoing resonance condition. The larger maximum intensity of the $2 \mathrm{LO}$ resonant profile compared to that of the $1 \mathrm{LO}$ arises from the phonon wavector $(\overrightarrow{\mathrm{q}})$ dependence of the electron-phonon Fröhlich interaction. ${ }^{22}$ In resonance, this interaction is

TABLE I. Lifetimes and intensities of the decay components of the 1LO mode around $34.4 \mathrm{meV}$ as a function of the excitation energy.

\begin{tabular}{llrlr}
\hline \hline Exc. $(\mathrm{eV})$ & $\tau_{d 1}(\mathrm{~ns}) \rightarrow$ Intensity $_{1}$ & \multicolumn{2}{c}{$\tau_{d 2}(\mathrm{ps}) \rightarrow$ Intensity $_{2}$} \\
\hline 1.160 & 1 & $30 \%$ & $\leq 30$ & $70 \%$ \\
1.136 & 1 & $55 \%$ & $\leq 30$ & $45 \%$ \\
1.110 & 1.1 & $70 \%$ & $\leq 30$ & $30 \%$ \\
1.089 & 1.1 & $85 \%$ & $\leq 30$ & $15 \%$ \\
1.059 & 1.3 & $55 \%$ & $\leq 30$ & $45 \%$ \\
\hline \hline
\end{tabular}


TABLE II. Temperature-dependent time-resolved photoluminescence of the LO mode at $34.6 \mathrm{meV}$ for $1.136 \mathrm{eV}$ of laser excitation. $\tau_{d 1}$ : long component

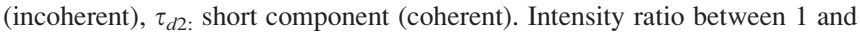
2. $\tau_{r}$ : rise time.

\begin{tabular}{lcccc}
\hline \hline$T(\mathrm{~K})$ & $\tau_{d 1}(\mathrm{~ns})$ & $\tau_{d 2}(\mathrm{ps})$ & Intensity $1: 2$ & $\tau_{r}(\mathrm{ps})$ \\
\hline 5 & 1 & $\leq 30$ & $50: 50$ & 300 \\
25 & 1 & $\leq 30$ & $50: 50$ & 300 \\
75 & 1.3 & $\leq 30$ & $67: 33$ & 330 \\
130 & 2.2 & $\leq 30$ & $85: 15$ & 290 \\
200 & 2 & - & - & 100 \\
\hline \hline
\end{tabular}

strongly enhanced leading to a maximum scattering probability for phonons fulfilling the condition $|\overrightarrow{\mathrm{q}}| a_{B} \approx 2$, where $a_{B}$ is the Bohr radius of the exciton. Consequently, the electron-phonon Fröhlich interaction (and the Raman intensity) will be larger for $2 \mathrm{LO}$ than for $1 \mathrm{LO}$ processes.

The temperature dependence of the phonon-assisted relaxation mechanisms was investigated by temperaturedependent time-resolved PL measurements for the 1LO transition at the excitation energy of $1.136 \mathrm{eV}$ (resonant with the first excited state). In Fig. 8 we show three representative transients for 5,75 , and $200 \mathrm{~K}$, while in Table II we show the resulting time constants as well as the intensity ratios between the incoherent $\left(\tau_{d 1}\right)$ and coherent components $\left(\tau_{d 2}\right)$. The intensity ratio $\mathrm{I}\left(\tau_{d 2}\right) / \mathrm{I}\left(\tau_{d 1}\right)$ decreases as the temperature increases toward $130 \mathrm{~K}$. For higher temperatures the coherent component could no longer be observed and the decay dynamics is governed by the long incoherent recombination. Concerning the temperature dependence of the lifetimes, $\tau_{d 2}$ remains independent of temperature within the experimental time resolution, while $\tau_{d 1}$ increases by a factor of 2 . The increases of $\tau_{d 1}$ can be understood by considering that as temperature increases so does the contribution of nonradiative mechanisms, such as Auger scattering or interaction with defects and impurities. Thereto, since the measured lifetimes are a combination of the radiative and nonradiative mechanisms $\left(1 / \tau_{\mathrm{i}}=1 / \tau_{\text {rad }}+1 / \tau_{\text {nrad }}\right)$, larger nonradiative lifetimes increase the effective measured lifetime. Furthermore, we have also observed a similar dependence for the lifetime of the $2 \mathrm{LO}$ (not shown), which reflects the fact that both transitions involve the same electronic states, i.e., the GS of the QDs electronic structure.

Finally, the temperature dependence observed for the rise component $\left(\tau_{\text {rise }}\right)$ provides a direct proof for the suggested phonon-bottleneck. This component remains approximately constant $(\approx 300 \mathrm{ps})$ below $130 \mathrm{~K}$, while for higher temperatures it strongly decreases $(\approx 100 \mathrm{ps})$. This temperature behavior resembles the temperature dependence of the GS intensity in Fig. 4, which increases toward a maximum around $130 \mathrm{~K}$. Therefore, the comparatively long rise time observed for low temperatures might originate in a phononbottleneck which is progressively smeared out as temperature increases. Furthermore, this weak phonon bottleneck is responsible for the observation of the coherent $\tau_{d 2}$ component. While for temperatures below $130 \mathrm{~K}$ this component is clearly observed, for higher temperatures it could not longer be resolved. Partly suppressed inhibition of the outgoing res- onance recombination through the GS allows the 1LO phonons to scatter coherently, i.e., the phonon-bottleneck controls the activation of the coherent component.

\section{SUMMARY}

Steady-state and time-resolved resonant photoluminescence measurements have been performed on an InAs/GaAs sample whose energy separation between the ground state and first excited state equals three times the energy of an InAs QD phonon mode. Besides the ground-state recombination, we observed two higher energy transitions located at the energy of 1LO/2LO phonons from the laser excitation, which originate from phonon-assisted processes. A strong contribution of these processes to the ground-state recombination was observed from the photoluminescence excitation spectra.

The temporal dynamics of these transitions provided a direct proof of the fact that, independently of the excitation mechanism, the electronic states involved in all the observed processes are those belonging to the ground state of the QDs electronic structure. Typical lifetimes of the ground state were around $1 \mathrm{~ns}$ for the low temperature range.

In addition, by investigating the temporal dynamics of the $1 \mathrm{LO}$ transition we were able to distinguish coherent from incoherent processes, which exhibited different typical lifetimes of around $30 \mathrm{ps}$ and $1 \mathrm{~ns}$, respectively. This picture is also supported by theoretical calculations using the densitymatrix formalism.

Finally, we have also shown that for temperatures below $130 \mathrm{~K}$ a phonon-bottleneck partly inhibits recombination of the ground state, whose intensity increases with increasing temperature toward $130 \mathrm{~K}$. This is also supported by the observation of a rise time of about 300 ps below $130 \mathrm{~K}$, which progressively vanishes for higher temperatures. Nevertheless, this effect does not dominate the ground-state recombination since the energy separation between the two first levels matched the energy of a $3 \mathrm{LO}$ and, consequently, the initially excited electrons can be successfully transferred to the ground state of the QDs.

\section{ACKNOWLEDGMENTS}

We acknowledge support from the Deutsche Forschungsgemeinschaft through Grant No. Sfb 787, Nanophotonik, and the Cluster of Excellence Unicat.

${ }^{1}$ Y. Arakawa and H. Sakaki, Appl. Phys. Lett. 40, 939 (1982).

${ }^{2}$ M. Asada, Y. Miyamoto, and Y. Suematsu, IEEE J. Quantum Electron. QE-22, 1915 (1986).

${ }^{3}$ Y. Arakawa and A. Yariv, IEEE J. Quantum Electron. QE-22, 1887 (1986).

${ }^{4}$ D. Bimberg, M. Grundmann, and N. N. Ledentsov, Quantum Dot Heterostructures (Wiley \& Sons, London, 1999).

${ }^{5}$ A. Lemaître, A. D. Ashmore, J. J. Finley, D. J. Mowbray, M. S. Skolnick, M. Hopkinson, and T. F. Krauss, Phys. Rev. B 63, 161309(R) (2001).

${ }^{6}$ S. Sanguineti, K. Wanatabe, T. Tateno, M. Wakaki, N. Koguchi, T. Kuroda, F. Minami, and M. Gurioli, Appl. Phys. Lett. 81, 613 (2002).

${ }^{7}$ O. Verzelen, R. Ferreira, and G. Bastard, Phys. Rev. B 62, R4809 (2000).

${ }^{8}$ E. Harbord, P. Spencer, E. Clarke, and R. Murray, Phys. Rev. B 80, 195312 (2009).

${ }^{9}$ S. Hameau, Y. Guldner, O. Verzelen, R. Ferreira, G. Bastard, J. Zeman, A. Lemaître, and J. M. Gerard, Phys. Rev. Lett. 83, 4152 (1999). 
${ }^{10} \mathrm{~S}$. Hameau, J. N. Isaia, Y. Guldner, E. Deleporte, O. Verzelen, R. Ferreira, and G. Bastard, J. Zeman, and J. M. Gerard, Phys. Rev. B 65, 85316 (2002).

${ }^{11}$ R. Heitz, I. Mukhametzhanov, O. Stier, A. Madhukar, and D. Bimberg, Phys. Rev. Lett. 83, 4654 (1999).

${ }^{12}$ M. Bissiri, G. B. Höger von Högersthal, A. S. Bhatti, M. Capizzi, A. Frova, P. Frigeri, and S. Franchi, Phys. Rev. B 62, 4642 (2000).

${ }^{13}$ O. Verzelen, R. Ferreira, and G. Bastard, Semicond. Sci. Technol. 19, S5 (2004).

${ }^{14}$ O. Verzelen, R. Ferreira, and G. Bastard, Phys. Rev. Lett. 88, 146803 (2002).

${ }^{15}$ R. Heitz, H. Born, A. Hoffmann, D. Bimberg, I. Mukhametzhanov, and A. Madhukar, Appl. Phys. Lett. 77, 3746 (2000).

${ }^{16}$ K. Mukai, N. Ohtsuka, H. Shoji, and M. Sugawara, Phys. Rev. B 54, R5243 (1996)
${ }^{17}$ R. Heitz, M. Veit, N. N. Ledentsov, A. Hoffmann, D. Bimberg, V. M. Ustinov, P. S. Kop'ev, and Zh. I. Alferov, Phys. Rev. B 56, 10435 (1997). ${ }^{18}$ S. Sanguinetti, M. Guzzi, E. Grilli, M. Gurioli, L. Seravalli, P. Frigeri, S. Franchi, M. Capizzi, S. Mazzuccato, and A. Polimeni, Phys. Rev. B 78, 085313 (2008).

${ }^{19}$ R. Heitz, M. Grundmann, N. N. Ledentsov, L. Eckey, M. Veit, D. Bimberg, V. M. Ustinov, A. Y. Egorov, A. E. Zhukov, P. S. Kop'ev, and Z. I. Alferov, Appl. Phys. Lett. 68, 361 (1996).

${ }^{20}$ S. Werner, P. Zimmer, A. Strittmatter, and A. Hoffmann, Mater. Res. Soc. Symp. Proc. 1053, EE03-03 (2008).

${ }^{21}$ J. Kabuss, S. Werner, A. Hoffmann, P. Hildebrandt, A. Knorr, and M. Richter, Phys. Rev. B 81, 075314 (2010).

${ }^{22}$ M. Cardona, "Resonance phenomena," in Light Scattering in Solids II, Topics in Applied Physics Vol. 50, edited by M. Cardona, G. Gäntherodt (Springer, Berlin, 1982), Chap. 2, pp. 19176. 